\title{
ON A CLASS OF AUTOMORPHIC FUNCTIONS*
}

BY

\section{J. I. HUTCHINSON}

In a paper entitled Ueber die Darstellung einiger Fälle der automorphen Primformen durch specielle Thetareihen, $\dagger$ BurkHaRdT has considered the monodromy group of the Riemann surface

$$
y^{3}=\left(x-a_{1}\right)\left(x-a_{2}\right)\left(x-\beta_{1}\right)^{2}\left(x-\beta_{2}\right)^{2},
$$

and has shown how a certain prime form which is automorphic for the group can be expressed by a theta series.

In the following article further results are given concerning this group and the functions belonging to it, the chief object in view being to obtain explicit analytic formulæ by means of which all functions of the group can be represented. To this end the theta-fuchsian functions of PoIncare are introduced and their expressions in terms of the hyperelliptic theta series deduced. It is then found that every function which is automorphic for the group considered can be expressed rationally in terms of the quotient of two particular theta-fuchsian functions.

\section{§1. The Riemann surface.}

The Riemann surface defined by $(1)$ is of genus $p=2$, and depends on one arbitrary constant. It is convenient to choose the two pairs of cross-cuts as indicated

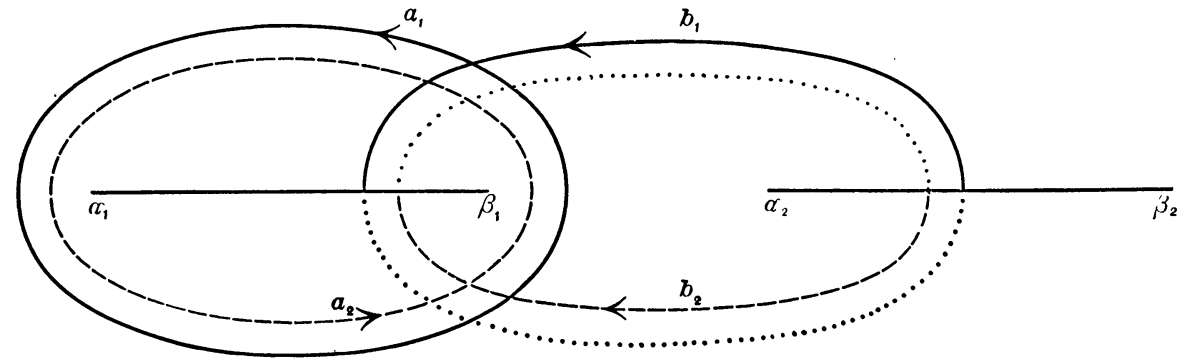

in the figure, where an unbroken line is used to represent a path in the first sheet, a broken line one in the second sheet and a dotted line one in the third sheet.

* Presented to the Society at the Ithaca meeting, August 19, 1901. Received for publication November 2, 1901.

† Mathematische Annalen, vol. 42 (1893), p. 185. See also articles by Hurwitz and RAUSENBERGER, in vol. 20 (1882), pp. 47, 125, 201. 
Two linearly independent integrals are

$$
u_{1}=\int \frac{d x}{y}, \quad u_{2}=\int \frac{\left(x-\beta_{1}\right)\left(x-\beta_{2}\right) d x}{y^{2}} .
$$

If for a particular value of $x$ the corresponding values of $y$ in the first, second, and third sheets of the surface are denoted by $y_{1}, y_{2}, y_{3}$ respectively, we shall assume $y_{2}=\rho y_{1}, y_{3}=\rho^{2} y_{2}$, where $\rho=\frac{1}{2}(-1+\sqrt{-3})$.

Denoting the moduli of periodicity of the integrals $u_{\nu}$ at the cuts $a_{1}, a_{2}$, $b_{1}, b_{2}$ by $A_{v 1}^{*}, A_{\nu 2}, B_{v 1}, B_{v 2}$ respectively, one sees easily from the above figure that

$$
A_{\nu 2}=-\rho^{\nu} A_{\nu 1}, \quad B_{\nu 2}=\rho^{2 \nu} B_{\nu 1}
$$

and derives the following table of periods.

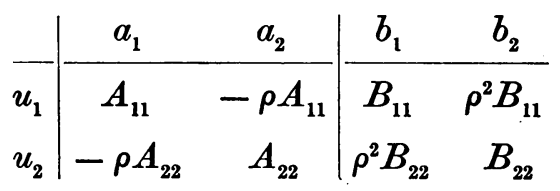

On account of the bilinear relations among the periods, these are subject to the condition,

$$
A_{11} B_{22}-A_{22} B_{11}=0 \text {. }
$$

The table of moduli for the normal integrals $v_{1}, v_{2}$ then assumes the form,

where

$$
\begin{array}{c|cc|cc}
- & a_{1} & a_{2} & b_{1} & b_{2} \\
v_{1} & \pi i & 0 & \frac{2 \pi i z}{\sqrt{3}} & -\frac{\pi i z}{\sqrt{3}} \\
v_{2} & 0 & \pi i & -\frac{\pi i z}{\sqrt{3}} & \frac{2 \pi i z}{\sqrt{3}}
\end{array}
$$

$$
z=i \rho^{2} \frac{B_{11}}{A_{11}}
$$

If we write $x+i y$ for $z$, the condition of inequality among the moduli is

$$
y>0 \text {. }
$$

\section{§2. Generation and properties of the group $G$.}

We now proceed to consider the effect on the moduli of a monodromy of the branch points. First, let $a_{1}$ move positively around $\beta_{1}$. Suppose that corresponding to this change the Riemann surface and its cross-cuts undergo a con- 
tinuous deformation. Using accents to distinguish the moduli of periodicity along the deformed cuts we have

whence

$$
\begin{aligned}
& A_{11}^{\prime}=A_{11}+(\rho-1) B_{11}, \\
& B_{11}^{\prime}=B_{11},
\end{aligned}
$$

$$
z^{\prime}=\frac{z}{1-\sqrt{3} z} \cdot
$$

In the second place interchange $a_{1}$ and $a_{2}$. This gives rise to the transformation

which is equivalent to

$$
A_{11}^{\prime}=-B_{11}, \quad B_{11}^{\prime}=-\rho^{2} A_{11},
$$

$$
z^{\prime}=-\frac{1}{z}
$$

It is easy to verify that any other monodromy of the branch points leads to a transformation which can be compounded of the two already obtained.

These two transformations generate a group of infinite order which will be denoted by $G$. It will be convenient to replace $\tau$ as a generating operation of the group by the substitution

$$
S=T \tau T, \text { that is, } z^{\prime}=z+\sqrt{3} .
$$

The transformation $S$ divides the $z$-plane into an infinite number of strips parallel to the $y$-axis and of width $\sqrt{ } \overline{3}$, while $T$ interchanges the outside and inside of the unit circle. Accordingly we may take as a fundamental region for the group $G$ a strip in the positive half plane bounded by the lines $x= \pm \frac{1}{2} \sqrt{3}$, and the unit circle.

Representing each transformation by the determinant of its coefficients, we have the following theorem.

Every operation of the group is of one of the two types:

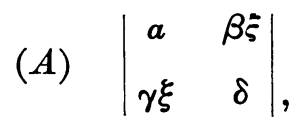

$$
\left|\begin{array}{cc}
a \xi & b \\
c & d \xi
\end{array}\right|
$$

where $\xi=\sqrt{3}$, and $a, b, \cdots, a, \beta, \cdots$ are integers satisfying the relations,

$$
\begin{aligned}
& 3 a d-b c=1, \\
& a \delta-3 \beta \gamma=1 .
\end{aligned}
$$

For, $S$ is of type $(A)$, and $T$ is of type $(B)$. Also, the product of two sub- 
stitutions of the same type gives one of type $(A)$ while the product of two of different types gives one of type $(B)$.

Conversely, every operation of type $(A)$, or of type $(B)$, is contained in $G$. For, starting with $(A)$, if $\beta$ is not positive and less than $|\delta|$, we apply $S^{m}$ and obtain

$$
\left|\begin{array}{cc}
a+3 m \gamma & (\beta+m \delta) \xi \\
\gamma \xi & \delta
\end{array}\right|=\left|\begin{array}{ll}
a^{\prime} & \beta^{\prime} \xi \\
\gamma \xi & \delta
\end{array}\right| .
$$

By properly choosing $m$ we can make $0<\beta^{\prime}<|\delta|$ since, on account of (4), $\beta$ and $\delta$ are relatively prime, and likewise $\beta^{\prime}$ and $\delta$.

Next, applying

we have

$$
T S^{n} T=\left|\begin{array}{ll}
-1 & 0 \\
n \xi & -1
\end{array}\right|
$$

$$
\left|\begin{array}{cc}
-a^{\prime} & -\beta^{\prime} \xi \\
\left(a^{\prime} n-\gamma\right) \xi & 3 n \beta^{\prime}-\delta
\end{array}\right|=\left|\begin{array}{cc}
-a^{\prime} & -\beta^{\prime} \xi \\
\gamma^{\prime} \xi & \delta^{\prime}
\end{array}\right| .
$$

By a proper choice of $n, \delta^{\prime}$ can be made positive and less than $3 \beta^{\prime}$.

There are now three possibilities to consider:

$$
\begin{aligned}
& \text { 1. } 0<\delta^{\prime}<\beta^{\prime}, \\
& \text { 2. } \beta^{\prime}<\delta^{\prime}<2 \beta^{\prime}, \\
& \text { 3. } 2 \beta^{\prime}<\delta^{\prime}<3 \beta^{\prime} .
\end{aligned}
$$

In case 1 we proceed as at first.

In case 2 by applying $S$ we obtain $\beta^{\prime \prime}=\delta^{\prime}-\beta^{\prime}$, which satisfies the condition

But since

$$
0<\beta^{\prime \prime}<\beta^{\prime}<\delta^{\prime} \text {. }
$$

it follows that

$$
\delta^{\prime}+\delta^{\prime}=2 \beta^{\prime}+2 \beta^{\prime \prime} \quad \text { and } \quad \delta^{\prime}<2 \beta^{\prime},
$$

$$
\delta^{\prime}>2 \beta^{\prime \prime} .
$$

If at the same time $\delta^{\prime}<3 \beta^{\prime \prime}$, case 3 is to be applied, otherwise we multiply by some power of $T S T$ as before.

In case 3 by applying $T S^{-1} T$ we get $\delta^{\prime \prime}\left(=3 \beta^{\prime}-\delta^{\prime}\right)$ with the condition

$$
0<\delta^{\prime \prime}<\beta^{\prime} \text {. }
$$

We can continue in this manner to reduce the numerical value of the $\beta$-coefficient until it reaches zero. The resulting substitution is a power of $T S T$.

Starting with an operation of type $(B)$ we first apply $T$. The result being then of type $(A)$ we proceed as above. 
§ 3. Transformation of the hyperelliptic thetas.

In order to obtain functions which are automorphic for the group $G$, we determine in the first place the effect of $S$ and $T$ upon the theta constants, that is, the theta functions with zero arguments and moduli $a_{j k}$, where

$$
a_{j j}=\frac{2 \pi i z}{\sqrt{3}}, \quad a_{12}=a_{21}=-\frac{\pi i z}{\sqrt{3}} .
$$

For this purpose we make use of the transformation theory of the theta functions. Let $b_{j k}$ be the new, or transformed moduli. Then for the substitution $S$,

where

$$
b_{j j}=\frac{2 \pi i z^{\prime}}{\sqrt{3}}, \quad b_{12}=-\frac{\pi i z^{\prime}}{\sqrt{3}},
$$

$$
z^{\prime}=z+\sqrt{ } 3
$$

The transformation formulæ for the $\vartheta$-functions require us to determine integral coefficients * $a_{j k}, \beta_{j k}, \gamma_{j k}, \delta_{j k}$ satisfying the identities

$$
\sum_{\kappa} b_{\nu \kappa}\left[a_{\kappa \mu} \pi i+\sum_{\lambda} \beta_{\kappa \lambda} a_{\mu \lambda}\right]=\pi i\left[\gamma_{\nu \mu} \pi i+\sum_{\lambda} \delta_{\nu \lambda} a_{\mu \lambda}\right],
$$

and subject also to certain bilinear relations. Substitute in this formula for $b_{\nu \kappa}$ and $a_{\mu \lambda}$ in terms of $z$, and equate coefficients of like powers of $z$ in both

\begin{tabular}{|c|c|c|c|}
\hline $\begin{array}{l}-m \\
n\end{array}$ & $\begin{array}{l}m+n \\
m\end{array}$ & 0 & \\
\hline$-2 m-n$ & $m+2 n$ & $-m$ & $n$ \\
\hline$m+2 n$ & $m-n$ & $m+n$ & $m$ \\
\hline
\end{tabular}
members. The final result is that

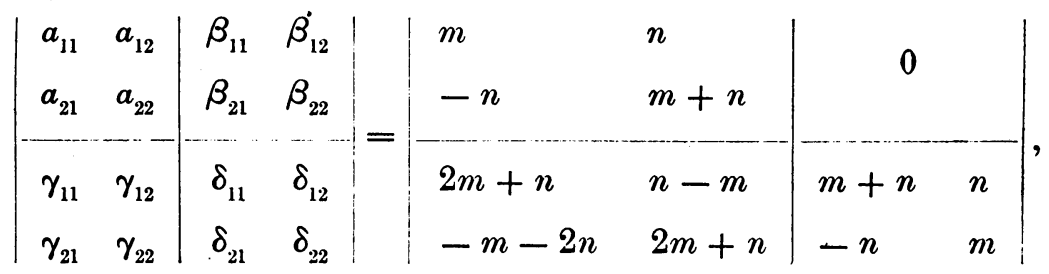

or

where $m, n$ are integers subject to the condition

$$
m^{2}+m n+n^{2}=1 \text {. }
$$

Let $\vartheta\left[\begin{array}{l}g \\ h\end{array}\right](z)$ denote a $\vartheta$-function of the first order with characteristic $\left[\begin{array}{l}g \\ h\end{array}\right]$,

* We use here the notation of Krazer and Prym, Neue Grundlagen, etc., p. 121. 
arguments zero, and moduli $a_{j k}$; also let $\vartheta\left[\begin{array}{l}g \\ h\end{array}\right]\left(z^{\prime}\right)$ be a similar function with moduli $b_{j k}$. Then one of the above transformations leads to the formula

$$
\vartheta\left[\begin{array}{l}
g \\
h
\end{array}\right]\left(z^{\prime}\right)=e^{-2 \pi i\left(g_{1}^{2}-g_{1} g_{2}+g_{2}^{2}\right)} \vartheta\left[\begin{array}{ll}
g_{1} \\
h_{1}+2 g_{1}-g_{2} & g_{2}^{2}+2 g_{2}-g_{1}
\end{array}\right](z) .
$$

Corresponding to $T$ we find in a similar manner the transformation

or

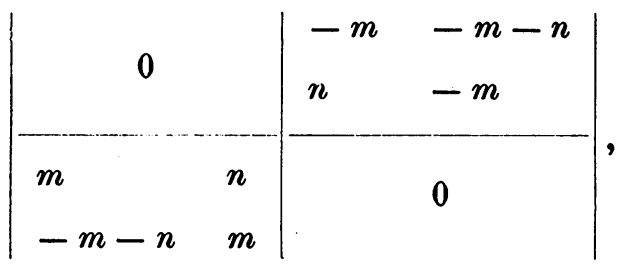

$$
\left|\begin{array}{ll|cc} 
& 0 & -m-n & -n \\
& -n & m \\
\hline m & n & 0
\end{array}\right|
$$

and the resulting $\vartheta$-formula

$$
\vartheta\left[\begin{array}{l}
g \\
h
\end{array}\right]\left(z^{\prime}\right)=-i z e^{2 \pi i \Sigma g_{j} h_{j}} \vartheta\left[\begin{array}{cc}
h_{2} & -h_{1} \\
-g_{2} & g_{1}
\end{array}\right](z) .
$$

It is to be remarked that (I) and (II) were obtained by making a particular choice for $m$ and $n$. Another choice for these numbers leads to different results. This indicates the existence of special relations among the $\vartheta$-constants which do not occur in the general case. These are conveniently obtained by considering the transformation for which $b_{j k}=a_{j k}$.

Computing the coefficients in the manner above explained we arrive at the result

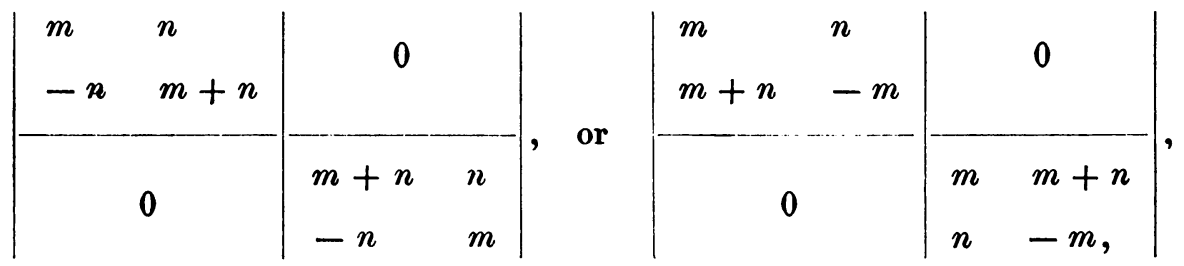

which leads to only two distinct relations, viz.,

$$
\begin{aligned}
& \vartheta\left[\begin{array}{l}
q \\
h
\end{array}\right](z)=\vartheta\left[\begin{array}{ll}
g_{2} & g_{1} \\
h_{2} & h_{1}
\end{array}\right](z), \\
& \vartheta\left[\begin{array}{l}
q \\
h
\end{array}\right](z)=\vartheta\left[\begin{array}{cc}
g_{1} \\
h_{1}+h_{2} & g_{1}-g_{2} \\
-h_{2}
\end{array}\right](z) .
\end{aligned}
$$


These formulæ reduce the $10 \vartheta$-constants with half integer characteristics to the four,*

$$
\vartheta\left[\begin{array}{c}
00 \\
00
\end{array}\right](z), \quad \vartheta\left[\begin{array}{c}
11 \\
00
\end{array}\right](0), \quad \vartheta\left[\begin{array}{c}
00 \\
11
\end{array}\right](z), \quad \vartheta\left[\begin{array}{c}
11 \\
11
\end{array}\right](z),
$$

which will be denoted by

$$
\vartheta, \quad \vartheta_{1}, \quad \vartheta_{2}, \quad \vartheta_{3}
$$

respectively. These four functions satisfy the identical relations,

$$
\begin{aligned}
& \vartheta^{2}+\vartheta_{3}^{2}=\vartheta_{1}^{2}+\vartheta_{2}^{2}, \\
& \vartheta^{4}-\vartheta_{3}^{4}=\vartheta_{1}^{4}+\vartheta_{2}^{4},
\end{aligned}
$$

which are readily deducible from the general relations among the thetas.

The effect of $S$ and $T$ on the functions (5) is shown in the following table.

\begin{tabular}{c|c|c|c|c} 
& $\vartheta$ & $\vartheta_{1}$ & $\vartheta_{2}$ & $\vartheta_{3}$ \\
\hline$S$ & $\vartheta$ & $-i \vartheta_{3}$ & $\vartheta_{2}$ & $-i \vartheta_{1}$ \\
$T$ & $-i z \vartheta$ & $-i z \vartheta_{2}$ & $-i z \vartheta_{1}$ & $-i z \vartheta_{3}$
\end{tabular}

Using $\kappa, \lambda, \mu$ (instead of the usual $\left.\kappa^{2}, \lambda^{2}, \mu^{2}\right)$ to denote the Rosenhain moduli we have, on account of (III),

whence

$$
\kappa=\frac{\vartheta_{1}^{2}}{\vartheta^{2}}, \quad \lambda=\frac{\vartheta_{3}^{2}}{\vartheta_{2}^{2}}, \quad \mu=\frac{\vartheta_{1}^{2} \vartheta_{3}^{2}}{\vartheta^{2} \vartheta_{2}^{2}}
$$

$$
\kappa \lambda=\mu=\frac{\lambda^{2}}{1-\lambda+\lambda^{2}},
$$

the second equality being deducible from (6).

\section{§4. The theta-fuchsian functions.}

We have now to show that every automorphic function belonging to $G$ can be expressed rationally in terms of any one of the Rosenhain moduli.

For this purpose we observe that the group $G$ is of genus zero, and hence it follows that every function belonging to $G$ can be expressed rationally in terms of any one which takes a given value once, and but once, in the fundamental region. In order to determine such functions, it is convenient to consider in this connection the theta-fuchsian functions introduced by PoINCARt.

A theta-fuchsian function $\Theta(z)$ is defined by the relation

$$
\Theta\left(z^{\prime}\right)=(\gamma z+\delta)^{2 m} \Theta(z),
$$

* In conformity to the customary notation the denominators of the characteristics are omitted. 
where

$$
z^{\prime}=\frac{a z+\beta}{\gamma z+\delta}
$$

is a substitution of the given group, and $m$ is a positive integer called the degree of the group. It is necessary to add that $\Theta(z)$ is one-valued, and in what follows we shall suppose that it has no discontinuities except on the $x$-axis.*

We have first of all to determine the number of zeros of $\Theta(z)$ in the fundamental region $R$. The two points $a=\frac{1}{2}(i-\sqrt{3}), a^{\prime}=\frac{1}{2}(i+\sqrt{3})$ are vertices of $R$, the first of which will be regarded as included in the fundamental region, and the other excluded from it. Starting now with $a$, we integrate $d \log \Theta(z)$ positively along the boundary of $R$, and along circular ares of infinitesimal radius excluding the regions in the vicinity of the vertices $a, a^{\prime}, b(=i)$, $c(=i \infty)$. The circular arc excluding $c$ is the straight line drawn from a point $z_{1}$, on the line $x=\frac{1}{2} \sqrt{3}$, parallel to the $x$-axis and ending in the point $z_{1}^{\prime}=z_{1}-\sqrt{ } \overline{3}$. The imaginary part of $z_{1}$ is regarded as indefinitely great.

Since $\Theta(z+\sqrt{3})=\Theta(z)$, we assume that $\Theta(z)$ can be expanded into a series of the form

where

$$
\begin{gathered}
\Theta(z)=\sum_{\kappa=n}^{\kappa \infty=} A_{\kappa} t^{\kappa}, \\
t^{\kappa}=e^{-\frac{2 \pi i z}{3}},
\end{gathered}
$$

and $n$ is a finite integer. If $n$ is negative $I$ shall say that $\Theta(c)$ is infinite of the $n$-th order; and, on the other hand, that it is zero of like order if $n$ is positive.

The line from $z_{1}$ to $z_{1}^{\prime}$ corresponds to the circumference of a circle about the origin in the $t$-plane, and generated by negative rotation from $\pi$ to $-\pi$. Hence

$$
\int_{z_{1}}^{z_{1}^{\prime}} d \log \Theta(z)=-2 n \pi i .
$$

Suppose in the next place that $z=a$ is a vertex of the fundamental region (or a congruent point) not on the $x$-axis, and let $p$ denote the period of the substitution having $a$ for fixed point. According to a theorem of Poincare's, $\dagger$ if

$$
m \equiv-q(\bmod p), \quad 0 \leqq q<p,
$$

$\Theta(z)$ can be expanded into a series of ascending powers of $z-a$ of the form

$$
A_{q}(z-a)^{q}+A_{q+p}(z-a)^{q+p}+\cdots,
$$

so that $z=a$ is a zero of order $q$ for every function of the same degree $m$.

$I$ wish at this point to distinguish between fixed and movable zeros of $\Theta(z)$. A fixed zero will be one which is common to all functions of the same degree $m$.

* Under this restriction we have what PoINCARÉ calls a function of the second species. In the present case a $\theta$-function having poles is the product of an automorphic function and a $\theta$-function of the second species, so that the above assumption is no restriction of generality.

† Acta Mathematica, vol. 1 (1882), p. 218. 
All others will be called movable zeros, since they change their positions in the $z$-plane when we pass from one $\Theta$-function to another.

In accordance with this distinction the point $a$ is a fixed zero of order $q$. If one, or more, of the coefficients $A_{q}, \ldots$ should vanish, we say that a corresponding number of movable zeros has coincided with the fixed zero, and that $a$ then counts as a fixed zero of order $q$, and a movable zero of order equal to the number of coefficients which have vanished.

On the supposition that $\Theta(c)$ is infinite of order $n$, we obtain for the number $M$ of movable zeros situated in the fundamental region $R$ the formula

$$
M=n+\frac{m}{3}-\frac{q_{a}}{6}-\frac{q_{b}}{2},
$$

where $q_{a}$ and $q_{b}$ are the values of $q$ at the two vertices $a$ and $b$ respectively.

If, on the other hand, $\Theta(z)$ becomes zero of order $n$ at $c$, we obtain for $M$ the value

$$
M=\frac{m}{3}-\frac{q_{a}}{6}-\frac{q_{h}}{2},
$$

counting the zero at $c$ as a movable zero of order $n$.

Restricting ourselves to the case in which $\Theta(z)$ is everywhere finite in $R$ (including $z=c$ ) we observe that $\Theta$-functions of degree $m=1$ do not exist, since for this value of $m$ formula $(10)$ gives $M=-1$. Further, $M=0$ when $m=2$, or 3 , and hence only one function of degree 2 , or 3 can exist. We see by reference to the table (7) that $\vartheta^{4}(z)$ is the $\Theta$-function of degree 2 .

Since $q_{a}=4, q_{b}=0$ when $m=2$, it follows that $\vartheta^{4}(z)$ has a fixed zero at $a$ (and all congruent points) of order 4 ; or, $\vartheta(z)$ is zero of the first order at these points, and nowhere else.

For $m=4, M=1$, we have two linearly independent functions,

$$
\Theta_{1}(z)=\vartheta^{8}, \quad \Theta_{2}(z)=\vartheta^{2} \vartheta_{1}^{2} \vartheta_{2}^{2} \vartheta_{3}^{2} .
$$

Every other $\Theta$-function of the same degree is expressible linearly in terms of these two. The quotient

$$
\frac{\Theta_{1}(z)}{\Theta_{2}(z)}=\eta(z)
$$

is an automorphic function which vanishes, and becomes infinite only once in the fundamental region, and hence takes any given value but once in the same region. Accordingly every function of the group can be expressed rationally in terms of $\eta(z)$. From (6) and (8) it readily follows that

$$
\eta(z)=\frac{\lambda^{2}(1-\lambda)^{2}}{\left(1-\lambda+\lambda^{2}\right)^{3}}=\kappa^{2}(1-\kappa)=\mu(1-\mu)^{2} .
$$


§5. Expression of the Rosenhain moduli in terms of the anharmonic ratio of the branch points.

It now remains to express the Rosenhain moduli in terms of $a_{1}, a_{2}, \beta_{1}, \beta_{2}$. This is accomplished by transforming the given hyperelliptic integrals into the Rosenhain normal form by means of a birational transformation. This requires the introduction of a new independent variable which is two-valued on the given Riemann surface. Such is the function

$$
\xi=\sqrt[3]{\frac{\left(x-\frac{\left.a_{1}\right)\left(x-a_{2}\right)}{\left(x-\beta_{1}\right)\left(x-\beta_{2}\right)}\right.}{\left(x-\beta_{1}\right)\left(x-\beta_{2}\right)}} .
$$

Differentiating, we obtain

where

$$
3 \xi^{2} d \xi=\frac{R d x}{\left(x-\beta_{1}\right)^{2}\left(x-\beta_{2}\right)^{2}},
$$

Hence

$$
R=\left(a_{2}-\beta_{2}\right)\left(x-a_{1}\right)\left(x-\beta_{1}\right)+\left(a_{1}-\beta_{1}\right)\left(x-a_{2}\right)\left(x-\beta_{2}\right) .
$$

$$
\frac{3\left(x-\beta_{1}\right)\left(x-\beta_{2}\right)}{R} d \xi=\frac{\left(x-\beta_{1}\right)\left(x-\beta_{2}\right)}{y^{2}} d x .
$$

Writing $\eta$ in the place of and integrating, we have *

$$
\frac{R}{\left(x-\beta_{1}\right)\left(x-\beta_{2}\right)}
$$

Similarly

$$
3 \int \frac{d \xi}{\eta}=u_{1}
$$

$$
3 \int \frac{\xi d \xi}{\eta}=u_{2} \text {. }
$$

The expression of $\eta$ in terms of $\xi$ gives the relation $\eta^{2}=\left(\beta_{1}-\beta_{2}\right)^{2} \xi^{6}+2\left[\left(a_{1}-\beta_{1}\right)\left(a_{2}-\beta_{2}\right)+\left(a_{1}-\beta_{2}\right)\left(a_{2}-\beta_{1}\right)\right] \xi^{3}+\left(a_{1}-a_{2}\right)^{2}$.

We find the branch points of the new surface by solving the equation $\eta=0$. Denoting these by $\xi_{i}(i=1,2, \ldots, 6)$, and writing

$$
\begin{gathered}
A=\left(a_{1}-\beta_{1}\right)\left(a_{2}-\beta_{2}\right)+\left(a_{1}-\beta_{2}\right)\left(a_{2}-\beta_{1}\right), \\
\Delta=A^{2}-\left(a_{1}-a_{2}\right)^{2}\left(\beta_{1}-\beta_{2}\right)^{2}=4\left(a_{1}-\beta_{1}\right)\left(a_{2}-\beta_{2}\right)\left(a_{1}-\beta_{2}\right)\left(a_{2}-\beta_{1}\right), \\
L=\left[\frac{-A+\sqrt{\Delta}}{\left(\beta_{1}-\beta_{2}\right)^{2}}\right]^{\frac{1}{3}}, \quad M=\left[\frac{-A-\sqrt{\Delta}}{\left(\beta_{1}-\beta_{2}\right)^{2}}\right]^{\frac{1}{3}},
\end{gathered}
$$

* Cf. Burkhardt, 1. c., p. 209. Burkhardt remarks that the hyperelliptic field is redacible to the elliptic by a transformation of higher degree. The transformation, which is of degree 2 , is given on page 14 of my Chicago dissertation, On the reduction of hyperelliptic furctions etc., Göttingen, 1897. 
we have

$$
\begin{array}{ll}
\xi_{1}=L, & \xi_{2}=\rho L, \\
\xi_{4}=M, & \xi_{5}=\rho^{2} L,
\end{array}
$$

If $\omega$ represents the anharmonic ratio

then

$$
\left[\beta_{1} a_{1} \beta_{2} a_{2}\right]=\frac{\left(a_{1}-\beta_{1}\right)\left(a_{2}-\beta_{2}\right)}{\left(a_{1}-\beta_{2}\right)\left(a_{2}-\beta_{1}\right)},
$$

$$
\frac{M}{L}=\left[\frac{1+\sqrt{\dot{\omega}}}{1-\sqrt{\omega}}\right]^{\frac{2}{3}}
$$

and the expressions for the RosenHaIn moduli take the form :

$$
\begin{aligned}
& \kappa=\left[\xi_{1} \xi_{5} \xi_{4} \xi_{2}\right]=\frac{L^{2}+L M+M^{2}}{3 L M}, \\
& \lambda=\left[\xi_{1} \xi_{3} \xi_{4} \xi_{2}\right]=\frac{M-\rho L}{L-\rho M}, \\
& \mu=\left[\xi_{1} \xi_{6} \xi_{4} \xi_{2}\right]=\frac{\left(L-\rho^{2} M\right)(M-\rho L)}{3 L M} .
\end{aligned}
$$

It is easy to verify that these expressions satisfy (9).

From the formulæ just deduced we easily find

$$
-\eta(z)=\kappa^{2}(\kappa-1)=\frac{16}{2} \overline{7} \frac{\omega}{(1-\omega)^{2}} .
$$

Hence, every automorphic function belonging to the group $G$ is rationally expressible in terms of $\omega, i$. e., in terms of the anharmonic ratio of the four branch points $a_{1}, a_{2}, \beta_{1}, \beta_{2}$. On the other hand it follows from the particular form of the expression in $\omega$ in (11) that every rational function of $\omega+\omega^{-1}$ is an automorphic function of the group.

Cornell University, August, 1901. 